\title{
Natural Characteristic of Thin-Wall Pipe under Uniformly Distributed Pressure
}

\author{
Chao-Feng $\mathrm{Li}^{1,2^{*}} \mathbb{C}$, Qian-Sheng Tang ${ }^{1}$, Hou-Xin She ${ }^{1}$ and Bang-Chun Wen ${ }^{1,2}$
}

\begin{abstract}
Natural characteristics of thin-wall pipe of the compressor under uniformly distributed pressure were presented in this paper based on a cylindrical shell model. In the traditional method, the beam model was usually used to analyze the pipe system. In actual fact, the pipe segment of the compressor was always broken in the form of a long crack or a partial hole and the phenomenon was hardly explained by beam model. According to the structure characteristic of compressor pipe segment, whose radius is large and thickness is little, shell model shows the advantage in this kind of pipe problem. Based on Sanders' shell theory, the vibration differential equation of pipe was established by applying the energy method. The influences of length to radius ratio $(L / R)$, thickness to radius ratio $(h / R)$, circumferential wave number ( $n$ ) and pressure ( $q$ ) on the natural frequencies of pipe were analyzed. The study shows: Pressure and structural parameters have a great effect on the natural characteristics of the pipe. Natural frequency increases as the pressure increases, especially for the higher mode. The sensitivity of natural frequency on pressure becomes stronger with $h / R$ ratio increases; when $L / R$ ratio is greater than a certain critical value, the influence of the pressure on natural frequency will no longer be obvious. The value of $n$ corresponding to the minimum natural frequency also depends on the value of pressure. In the end, analysis of the forced vibration of a specific pipeline model was given and the modal shapes were illustrated to understand the break of the pipe. The research here will provide the theory support for the dynamic design of related pressure pipe and further experiment study should be employed.
\end{abstract}

Keywords: Pipe vibration, Natural frequency, Pulse pressures, Geometric parameters, Modal shape

\section{Introduction}

Pipe is widely used in many engineering applications, in which the centrifugal compressor is a kind of large equipment, which is widely used in the field of oil, chemical, and so on. Due to the characteristics of compressing work, pulse pressures with high frequency are loaded to the inlet/outlet and the distal bending, causing the highfrequency vibration of the pipe, whose main characteristic is the partial vibration of the wall of the pipe. The high-frequency vibration has a great threat to the production safety. Strong vibration may cause pipe broken, chemical gas leak and even an explosion. So the study of pipe vibration has an important signification.

For the pipe vibration, beam model and shell model are usually used to get numerical or analytical solutions [1].

\footnotetext{
*Correspondence: chfli@mail.neu.edu.cn

${ }^{1}$ School of Mechanical Engineering \& Automation, Northeastern University, Shenyang 110819, China

Full list of author information is available at the end of the article
}

In open literature, beam model was usually applied to the pipe system, especially fluid-conveying pipe. For example, Kheiri et al. [2,3] modeled long pipes towed underwater as a uniform, neutrally buoyant Euler-Bernoulli beam. External force and boundary conditions were also simply modeled to obtain the partial differential equations of the motion. A finite difference technique was used to get the results which showed that a long pipe may lose stability due to higher flow velocities by flutter and divergence and the deformation may happen close to the downstream end. Ghayesh et al. [4] studied the vibration of cantilevered pipe conveying fluid and the nonlinear planar dynamic response was investigated by bifurcation diagrams of the system vis flow velocity and other kinetics characteristics. Yamashita et al. [5] used theoretically analysis and experimental verification to study the selfexcited nonplanar motion of the vertical cantilevered pipe subjected the horizontal external excitation. Zhang et al. $[6,7]$ studied the internal resonance and external 
resonance of pipe conveying fluid in the supercritical flow speed through the analytical results and the numerical integrations.

Beam model is often applied when both length to radius ratio and thickness are large. However, the structural features of the compressor pipe are the little length to radius ratio and the little thickness. It is a thin-wall structure and if it suffers a high-frequency cyclic loading, the highfrequency vibration will be aroused. Vibration damage of the pipe outlet such as a long crack or a partial hole may happen which was mainly caused by fatigue fracture due to resonance. And the broken form of pipe may not be fully explained based on beam model. Therefore, due to the particularity of the research object, the shell model was introduced. For the shell vibration, many works for the shell model have been done [8]. Lessia et al. [9] developed a series of solution techniques for the complicated shell structures with various boundary conditions based on various shell theories, among which Donnell theory, Flugge's theory, Love's theory, and Sanders' theory are most widely used. In recent decades, many accurate and efficient methods have been developed to determine the vibration characteristics which include natural frequencies, modes, and so on. Refs. [10, 11] used the method of the generalized differential quadrature (GDQ) to solve the vibration of cylindrical shells. After then, they analyzed the vibration of functionally graded cylindrical shells with Ritz method [12-14]. Xie et al. [15] presented a novel and efficient solution for free vibrations of thin cylindrical shells subjected to various boundary conditions by using the Haar wavelet discretization method based on the Goldenveizer-Novozhilov shell theory. Xie et al. [16] applied Transfer matrix method to analyze free vibration of circular cylindrical shells based on the Flugge's shell theory and analyzed the natural frequencies and natural modes. In calculation, they used the integral method instead of the Runge-Kutta method to obtain more accurate numerical results. Dai et al. [17] used the elastic equations to obtain an exact series solution for the vibration analysis of circular cylindrical shells with arbitrary boundary conditions in which a Fourier series and auxiliary functions were used to represent the three displacements, and it is easy to deal with complicated boundary conditions using this method. Based on Sanders' theory, Sun et al. [18] studied the vibration studies of rotating cylindrical shells with arbitrary edges using characteristic orthogonal polynomials in the Rayleigh-Ritz method. Isvandzibaei et al. [19, 20] studied the vibration of multiple layered cylindrical shells under lateral pressure with asymmetric boundary conditions.

To sum up, numerous studies on pipe and shell have been published. However, those studies based on the engineering background of the pressure pipe of the centrifugal compressor are relatively fewer. So for the pressure pipe of the centrifugal compressor, free and forced vibration problems should be further discussed. This paper presented the study on the natural characteristics of pressure pipe with SS-SS boundary condition using cylindrical shell model. Based on Sanders' shell theory, the vibration differential equation was obtained by using the energy method. The displacement shape functions were assumed by beam functions in the axial direction and the trigonometric functions in the circumferential direction. The influences of length to radius ratio, thickness to radius ratio, circumferential wave number and pressure on natural characteristics were analyzed. In the end, the steady responses of the forced vibration for a specific pipe model were obtained and the modal shapes were illustrated to understand the break of the pipe. And the results would be signification for the design of the pressure pipe in the centrifugal compressor.

\section{Theoretical Formulations}

\subsection{Kinetic Energy and Potential Energy of Pressure Pipe}

Pressure pipe which is regarded as a cylindrical shell is shown in Figure 1. In the figure, the thickness, the length and the mean radius of the cylinder are denoted by $h, L$ and $R$, respectively. As the movement of each point on the pipe can be expressed in the movement of the points on the middle surface of the pipe segment, the middle surface is only considered. Assuming that the pipe ends are governed by simply-supported boundary conditions, the cylindrical coordinate is established, where $x, \theta$, and $z$ represent the axial direction, circumferential direction and the radial direction, respectively. The components of the deformation of the cylindrical shell with references to this coordinate system are denoted by $u, v$ and $w$ in the $x, y$ and $z$ directions. The pipe is under uniform distributed pressure $q$. The material of the pipe is assumed to be isotropic with the mass density $\rho$, Poisson's ratio $\mu$, and Young's modulus $E$.

Setting $\xi=x / L$, the kinetic energy for vibration of a pipe can be written as:

$$
T=\frac{\rho h L}{2} \int_{0}^{1} \int_{0}^{2 \pi}\left(\left(\frac{\partial u}{\partial t}\right)^{2}+\left(\frac{\partial v}{\partial t}\right)^{2}+\left(\frac{\partial w}{\partial t}\right)^{2}\right) R \mathrm{~d} \xi \mathrm{d} \theta,
$$

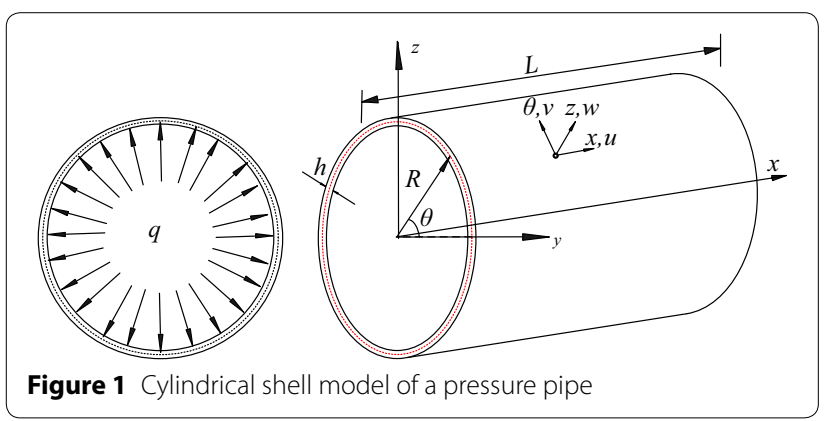


Based on Sanders' shell theory, the strains are given by:

$$
\varepsilon_{x}=\varepsilon_{x}^{0}+z \chi_{x}, \quad \varepsilon_{\theta}=\varepsilon_{\theta}^{0}+z \chi_{\theta}, \quad \varepsilon_{x \theta}=\varepsilon_{x \theta}^{0}+z \chi_{x \theta} .
$$

where the middle surface strain and the middle surface curvature are defined as:

$$
\begin{aligned}
& \varepsilon_{x}^{0}=\frac{\partial u}{L \partial \xi}, \quad \varepsilon_{\theta}^{0}=\frac{\partial v}{R \partial \theta}+\frac{w}{R}, \quad \varepsilon_{x \theta}^{0}=\frac{\partial u}{R \partial \theta}+\frac{\partial v}{L \partial \xi} . \\
& \left\{\begin{array}{l}
\chi_{x}=-\frac{\partial^{2} u}{L^{2} \partial \xi \xi^{2}} \\
\chi_{\theta}=\frac{\partial v}{R^{2} \partial \theta}-\frac{\partial^{2} w}{R^{2} \partial \theta^{2}} \\
\chi_{x \theta}=-\frac{\partial u}{2 R^{2} \partial \theta}+\frac{3 \partial v}{2 R L \partial \xi}-\frac{2 \partial^{2} w}{R L \partial \xi \partial \theta}
\end{array}\right.
\end{aligned}
$$

The stress-strain relationship of pipe can be written as:

$$
\left\{\begin{array}{c}
\sigma_{x} \\
\sigma_{\theta} \\
\tau_{x \theta}
\end{array}\right\}=\left[\begin{array}{lll}
Q_{11} & Q_{12} & \\
Q_{21} & Q_{22} & \\
& & Q_{33}
\end{array}\right]\left\{\begin{array}{c}
\varepsilon_{x} \\
\varepsilon_{\theta} \\
\varepsilon_{x \theta}
\end{array}\right\} .
$$

where

$$
\begin{aligned}
Q_{11}=Q_{22} & =\frac{E}{1-\mu^{2}}, \quad Q_{12}=Q_{21}=\frac{\mu E}{1-\mu^{2}}, \\
Q_{33} & =\frac{E}{2(1+\mu)} .
\end{aligned}
$$

The strain energy of pipe $U_{\varepsilon}$ is:

$$
U_{\varepsilon}=\frac{1}{2} \int_{V} \sigma_{x} \varepsilon_{x}+\sigma_{\theta} \varepsilon_{\theta}+\tau_{x \theta} \varepsilon_{x \theta} \mathrm{d} V
$$

Considering a pipe under uniform distributed pressure, the potential energy of the pipe due to this initial distributed pressure can be derived [20], that is

$$
U_{q}=-\int_{0}^{1} \int_{0}^{2 \pi} \frac{q L}{2}\left[\left(\frac{\partial^{2} w}{\partial \theta^{2}}+w\right) w\right] \mathrm{d} \theta \mathrm{d} \xi,
$$

The total potential energy for vibration of a pipe under uniform distributed pressure is:

$$
U=U_{\varepsilon}+U_{q} .
$$

\subsection{Galerkin Discretization of the Energy Equation}

In order to use the Rayleigh-Ritz method, the displacements $u, v$ and $w$ should be expressed in terms of generalized coordinates:

$$
\left\{\begin{aligned}
u(\xi, \theta, t) & =\sum_{m=1}^{i} \sum_{n=1}^{j} U_{m n}(\xi, \theta) q_{m n}^{u}(t)=\boldsymbol{U}^{\mathrm{T}} \boldsymbol{q}_{U}(t), \\
v(\xi, \theta, t) & =\sum_{m=1}^{i} \sum_{n=1}^{j} V_{m n}(\xi, \theta) q_{m n}^{v}(t)=\boldsymbol{V}^{\mathrm{T}} \boldsymbol{q}_{V}(t), \\
w(\xi, \theta, t) & =\sum_{m=1}^{i} \sum_{n=1}^{j} W_{m n}(\xi, \theta) q_{m n}^{w}(t)=W^{\mathrm{T}} \boldsymbol{q}_{W}(t) .
\end{aligned}\right.
$$

where $\boldsymbol{q}_{U}, \boldsymbol{q}_{V}$, and $\boldsymbol{q}_{W}$ are the generalized coordinates or modal coordinates, and $\boldsymbol{U}, \boldsymbol{V}$, and $\boldsymbol{W}$ are the displacement shape functions or the principal vibration modes which must satisfy the geometric boundary conditions. They are written by

$$
\left\{\begin{array}{l}
\boldsymbol{U}=\left[U_{11}, \cdots, U_{1 j}, U_{21}, \cdots, U_{2 j}, \cdots, U_{m n}, \cdots, U_{i 1}, \cdots, U_{i j}\right]^{\mathrm{T}}, \\
\boldsymbol{V}=\left[V_{11}, \cdots, V_{1 j}, V_{21}, \cdots, V_{2 j}, \cdots, V_{m n}, \cdots, V_{i 1}, \cdots, V_{i j}\right]^{\mathrm{T}}, \\
\boldsymbol{W}=\left[W_{11}, \cdots, W_{1 j}, W_{21}, \cdots, W_{2 j}, \cdots, W_{m n}, \cdots, W_{i 1}, \cdots, W_{i j}\right]^{\mathrm{T}}, \\
\boldsymbol{q}_{U}=\left[q_{11}^{u}, \cdots, q_{1 j}^{u}, q_{21}^{u}, \cdots, q_{2 j}^{u}, \cdots, q_{m n}^{u}, \cdots, q_{i 1}^{u}, \cdots, q_{i j}^{u}\right]^{\mathrm{T}}, \\
\boldsymbol{q}_{V}=\left[q_{11}^{v}, \cdots, q_{1 j}^{v}, q_{21}^{v}, \cdots, q_{2 j}^{v}, \cdots, q_{m n}^{v}, \cdots, q_{i 1}^{v}, \cdots, q_{i j}^{v}\right]^{\mathrm{T}}, \\
\boldsymbol{q}_{W}=\left[q_{11}^{w}, \cdots, q_{1 j}^{w}, q_{21}^{w}, \cdots, q_{2 j}^{w}, \cdots, q_{m n}^{w}, \cdots, q_{i 1}^{w}, \cdots, q_{i j}^{w}\right]^{\mathrm{T}} .
\end{array}\right.
$$

Then the kinetic energy and potential energy are expressed in terms of the generalized coordinates and displacement shape functions. Substituting Eq. (10) and Eq. (11) into Eq. (1), we can obtain

$$
T=\frac{1}{2} \dot{\boldsymbol{q}}_{U}^{\mathrm{T}} \boldsymbol{M}_{1} \dot{\boldsymbol{q}}_{U}+\frac{1}{2} \dot{\boldsymbol{q}}_{V}^{\mathrm{T}} \boldsymbol{M}_{2} \dot{\boldsymbol{q}}_{V}+\frac{1}{2} \dot{\boldsymbol{q}}_{W}^{\mathrm{T}} \boldsymbol{M}_{3} \dot{\boldsymbol{q}}_{W},
$$

where $\boldsymbol{M}_{1}, \boldsymbol{M}_{2}$, and $\boldsymbol{M}_{3}$ are the modal mass matrices and they are listed in Appendix.

Substituting Eqs. (3)-(8) and (10), (11) into Eq. (9), we can obtain

$$
\begin{aligned}
U & =\frac{1}{2} \boldsymbol{q}_{U}^{\mathrm{T}} \boldsymbol{K}_{1} \boldsymbol{q}_{U}+\frac{1}{2} \boldsymbol{q}_{U}^{\mathrm{T}} \boldsymbol{K}_{2} \boldsymbol{q}_{V}+\frac{1}{2} \boldsymbol{q}_{U}^{\mathrm{T}} \boldsymbol{K}_{3} \boldsymbol{q}_{W} \\
& +\frac{1}{2} \boldsymbol{q}_{V}^{\mathrm{T}} \boldsymbol{K}_{4} \boldsymbol{q}_{V}+\frac{1}{2} \boldsymbol{q}_{V}^{\mathrm{T}} \boldsymbol{K}_{5} \boldsymbol{q}_{W}+\frac{1}{2} \boldsymbol{q}_{W}^{\mathrm{T}}\left(\boldsymbol{K}_{6}+\boldsymbol{H}\right) \boldsymbol{q}_{W} .
\end{aligned}
$$

where $\boldsymbol{K}_{1}, \boldsymbol{K}_{2}, \boldsymbol{K}_{3}, \boldsymbol{K}_{4}, \boldsymbol{K}_{5}, \boldsymbol{K}_{6}$ and $\boldsymbol{H}$ are the modal stiffness matrices, which are also presented in Appendix. 


\subsection{Differential Equation of Forced Vibration}

Considering the case of damping, the form of Lagrange equations is expressed as

$$
\frac{\mathrm{d}}{\mathrm{d} t}\left(\frac{\partial T_{b}}{\partial \dot{\boldsymbol{q}}}\right)-\frac{\partial T_{b}}{\partial \boldsymbol{q}}+\frac{\partial U_{b}}{\partial \boldsymbol{q}}=\boldsymbol{F}+\boldsymbol{F}_{\boldsymbol{c}}
$$

where $\boldsymbol{F}$ is forcing matrix and $\boldsymbol{F}_{\mathrm{c}}$ is damping force matrix. According to the type as Eq. (14), the Lagrange equations can also be written by:

$$
\left\{\begin{array}{l}
\frac{\mathrm{d}}{\mathrm{d} t}\left(\frac{\partial T}{\partial \dot{\boldsymbol{q}}_{U}}\right)-\frac{\partial T}{\partial \boldsymbol{q}_{U}}+\frac{\partial U}{\partial \boldsymbol{q}_{U}}=\boldsymbol{F}_{U q}+\boldsymbol{F}_{U c}, \\
\frac{\mathrm{d}}{\mathrm{d} t}\left(\frac{\partial T}{\partial \dot{\boldsymbol{q}}_{V}}\right)-\frac{\partial T}{\partial \boldsymbol{q}_{V}}+\frac{\partial U}{\partial \boldsymbol{q}_{V}}=\boldsymbol{F}_{V q}+\boldsymbol{F}_{V c}, \\
\frac{\mathrm{d}}{\mathrm{d} t}\left(\frac{\partial T}{\partial \dot{\boldsymbol{q}}_{W}}\right)-\frac{\partial T}{\partial \boldsymbol{q}_{W}}+\frac{\partial U}{\partial \boldsymbol{q}_{W}}=\boldsymbol{F}_{W q}+\boldsymbol{F}_{W c} .
\end{array}\right.
$$

Substituting Eqs. (12) and (13) into Eq. (15), the equation of motion of the pipe can be obtained as

$$
\boldsymbol{M} \ddot{\boldsymbol{q}}+\boldsymbol{C} \dot{\boldsymbol{q}}+\boldsymbol{K} \boldsymbol{q}=\boldsymbol{F} .
$$

Then the generalized mass matrix, the generalized damping matrix, stiffness matrix, forcing matrix, and the generalized coordinates can be written by

$$
\left\{\begin{array}{l}
\boldsymbol{M}=\left[\begin{array}{ccc}
\boldsymbol{M}_{1} & \mathbf{0} & \mathbf{0} \\
\mathbf{0} & \boldsymbol{M}_{2} & \mathbf{0} \\
\mathbf{0} & \mathbf{0} & \boldsymbol{M}_{3}
\end{array}\right], \\
\boldsymbol{C}=\left[\begin{array}{ccc}
\boldsymbol{C}_{e q 1} & \mathbf{0} & \mathbf{0} \\
\mathbf{0} & \boldsymbol{C}_{e q 2} & \mathbf{0} \\
\mathbf{0} & \mathbf{0} & \boldsymbol{C}_{e q 3}
\end{array}\right], \\
\boldsymbol{K}=\left[\begin{array}{ccc}
\boldsymbol{K}_{1} & \frac{1}{2} \boldsymbol{K}_{2} & \frac{1}{2} \boldsymbol{K}_{3} \\
\frac{1}{2} \boldsymbol{K}_{2}^{\mathrm{T}} & \boldsymbol{K}_{4} & \frac{1}{2} \boldsymbol{K}_{5} \\
\frac{1}{2} \boldsymbol{K}_{3}^{\mathrm{T}} & \frac{1}{2} \boldsymbol{K}_{5}^{\mathrm{T}} & \boldsymbol{K}_{6}+\boldsymbol{H}
\end{array}\right], \\
\boldsymbol{F}=\left[\begin{array}{l}
\boldsymbol{F}_{U q} \\
\boldsymbol{F}_{V q} \\
\boldsymbol{F}_{W q}
\end{array}\right], \boldsymbol{q}=\left[\begin{array}{l}
\boldsymbol{q}_{U} \\
\boldsymbol{q}_{V} \\
\boldsymbol{q}_{W}
\end{array}\right] .
\end{array}\right.
$$

where the generalized damping matrix $C$ can be gotten by

$$
\left\{\boldsymbol{C}_{e q 1}, \boldsymbol{C}_{e q 2}, \boldsymbol{C}_{e q 3}\right\}=\int_{A} c_{e q}\left\{\boldsymbol{U}^{\mathrm{T}} \boldsymbol{U}, \boldsymbol{V}^{\mathrm{T}} \boldsymbol{V}, \boldsymbol{W}^{\mathrm{T}} \boldsymbol{W}\right\} \mathrm{d} A .
$$

\subsection{Differential Equation of Forced Vibration with SS-SS \\ Boundary Conditions}

The principal mode shapes of a cylindrical shell with SS-SS boundaries $U, V, W$, and the items about time $q_{U}$, $q_{V}, q_{W}$ can be expressed as

$$
\left\{\begin{array}{l}
U_{m n}(\xi, \theta)=\cos (m \pi \xi) \cos (n \theta) \\
V_{m n}(\xi, \theta)=\sin (m \pi \xi) \sin (n \theta) \\
W_{m n}(\xi, \theta)=\sin (m \pi \xi) \cos (n \theta)
\end{array}\right.
$$

$$
\left\{\begin{array}{l}
q_{U}=A_{m n}^{x} \cos (\omega t), \\
q_{V}=B_{m n}^{\theta} \cos (\omega t), \\
q_{W}=C_{m n}^{z} \cos (\omega t),
\end{array}\right.
$$

where $m=1,2,3, \ldots, i, n=1,2,3, \ldots, j . m$ is the half wave in the axial direction and $n$ is the wave in the circumferential direction. $\omega_{m n}$ is the circular frequency of cylindrical pipe. Under the modal $(m, n)$, pipe vibration Eq. (16) is three degrees of freedom, and $\boldsymbol{M}, \boldsymbol{C}, \boldsymbol{K}$ is three order matrix. Substituting Eqs. (19), (20) into Eq. (16), then Eq. (16) can be changed as:

$$
\frac{\pi}{2} \rho h L R \boldsymbol{I}_{3 \times 3} \ddot{\boldsymbol{q}}+\frac{\pi}{2} R L c_{e q} \boldsymbol{I}_{3 \times 3} \dot{\boldsymbol{q}}+\frac{\pi}{2} \boldsymbol{K}_{0} \boldsymbol{q}=\boldsymbol{F} .
$$

Ignoring the system damping and generalized force, the general solution of Eq. (21) can be written as

$$
\boldsymbol{q}(t)=\boldsymbol{q}_{0} e^{f t},
$$

where $\boldsymbol{q}_{0}$ is the eigenvector and $f$ is the eigenvalue. Substituting Eq. (22) into the homogeneous differential equation of Eq. (21), the eigenvalue problem is gotten:

$$
\left(\rho h L R f^{2} \boldsymbol{I}_{3 \times 3}+\boldsymbol{K}_{0}\right) \boldsymbol{q}_{0}=0,
$$

from which the eigenvectors and eigenvalues can be obtained. The imaginary parts of the eigenvalues are the natural frequencies of the pipe. The smallest of the roots is the natural frequency of interest in this paper. The frequency parameter can be obtained by:

$$
\Omega_{m n}=f_{m n} R \sqrt{\rho\left(1-\mu^{2}\right) / E} .
$$


Table 1 Comparison of frequency parameters for the cylindrical shell without pressure with SS-SS boundary condition $(m=1, L / R=20, h / R=0.02, \mu=0.3)$

\begin{tabular}{llll}
\hline $\boldsymbol{n}$ & Eq. (24) & Ref. [9]/[10] & Error [9]/[10] (\%) \\
\hline 1 & 0.016101 & $0.016101 / 0.016102$ & $0.00 / 0.01$ \\
2 & 0.009377 & $0.009382 / 0.009387$ & $0.05 / 0.00$ \\
3 & 0.022103 & $0.022105 / 0.022108$ & $0.01 / 0.02$ \\
4 & 0.042094 & $0.042095 / 0.042096$ & $0.00 / 0.00$ \\
5 & 0.068007 & $0.068008 / 0.068008$ & $0.00 / 0.00$ \\
6 & 0.099729 & $0.099730 / 0.099730$ & $0.00 / 0.00$ \\
7 & 0.137238 & $0.137239 / 0.137239$ & $0.00 / 0.00$ \\
8 & 0.180527 & $0.180527 / 0.180527$ & $0.00 / 0.00$ \\
9 & 0.229593 & $0.229594 / 0.229594$ & $0.00 / 0.00$ \\
10 & 0.284435 & $0.284435 / 0.284435$ & $0.00 / 0.00$ \\
\hline
\end{tabular}

\section{Model Validation}

In order to validate the accuracy of the model developed, the results of the cylindrical shell without pressure $(q=0)$ are compared with those in the open literature, which are shown in Table 1 . The frequency parameters in Refs. [2,3] were obtained by different methods based on Love's shell theory. What can be found is that the differences between the frequency parameters presented in this paper and those from the references are very small. Except the first maximum percentage difference $0.05 \%$, the errors are less than $0.02 \%$, including the major zero error. Therefore, the model and method used in this paper have high accuracy and credibility and provide the theoretical basis for the following calculation.

\section{Results and Discussion}

In this section, the pipe is subjected to uniformly distributed pressure with SS-SS boundary conditions. $E=2.06 \times 10^{11} \mathrm{~Pa}, \rho=7850 \mathrm{~kg} / \mathrm{m}^{3}$ and $\mu=0.3$. The influences of thickness to radius ratio $(h / R)$, length to radius ratio $(L / R)$, circumferential wave number $(n)$ and uniformly distributed pressure $(q)$ on natural frequencies of pipe were studied.

\subsection{Influence of Pressure and $h / R$ on Natural Frequencies}

The natural frequencies are shown in Figures 2 and 3 were calculated for $m=1, n=6$, and $L / R=4$.

Figure 2 shows the variation of the natural frequency of the pipe for different $h / R$ ratios with uniformly distributed pressure $(q)$. As demonstrated, when $q=0$, the natural frequency increases as the value of $h / R$ increases. When $q>0$, the natural frequency initially decreases and then increases, the trend of growth is more obvious when

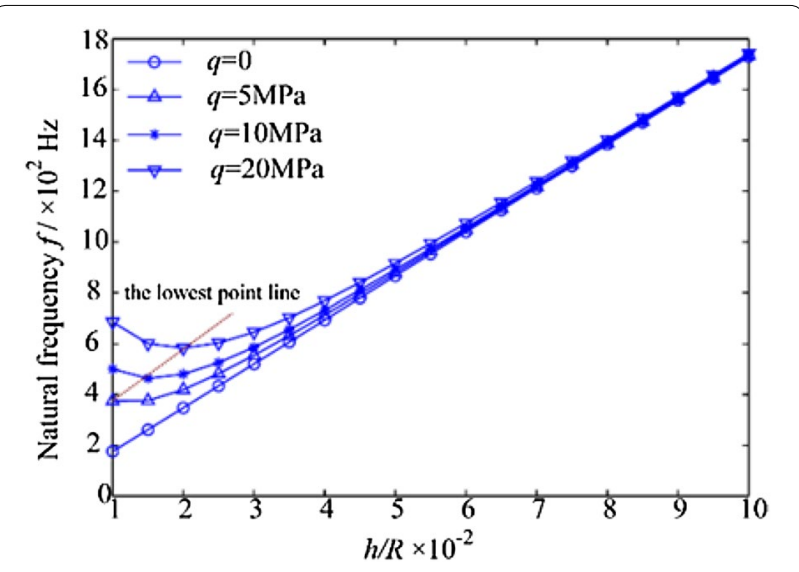

Figure 2 Variation of the natural frequency against $h / R$ ratio for several uniformly distributed pressures

$q$ becomes larger. All of these graphs show that the point of the minimum value of natural frequency moves to the right when $q$ increases. When $q=5 \mathrm{MPa}$, it appears beside $h / R=0.01$. When $q=10 \mathrm{MPa}$, it appears beside $h / R=0.015$. And when $q=20 \mathrm{MPa}$, it appears beside $h / R=0.02$. All express that the lowest point of the curve moves to the right when $q$ increases (shown by the connection line of the lowest point in the figure). And when $h / R$ ratio increases to a certain extent, the curve of growth of natural frequency almost becomes a straight line.

Figure 3 shows the variation of the natural frequency of the pipe against different $q$ with several special values of $h / R$ radio. As demonstrated, the natural frequencies increase as the value of $q$ increases, and the effect is more obvious when $h / R$ is 0.01 and 0.02 . The curve of variation of the natural frequency at $h / R=0.01$ intersects the one with $h / R=0.02$ due to the larger effect of $q$ on the natural frequency at $h / R=0.01$. Therefore, the sensitivity of $q$ on natural frequency for thin wall pipe is stronger than the sensitivity of $q$ for thick wall pipe. In addition, what can be seen is that the curve of variation of natural frequency flattens out when $h / R \geq 0.04$. At the same time, the effect of $q$ on natural frequency becomes insensitive.

\subsection{Influence of Pressure and $L / R$ Radio on Natural Frequencies}

The natural frequencies are shown in Figures 4 and 5 were calculated for $m=1, n=6$, and $h / R=0.02$.

Figure 4 shows the variation of the natural frequency of the pipe for different $L / R(1<L / R<20)$ with uniformly distributed pressure $(q)$. If $q$ is a constant value, the natural frequency decreases as the value of $L / R$ 


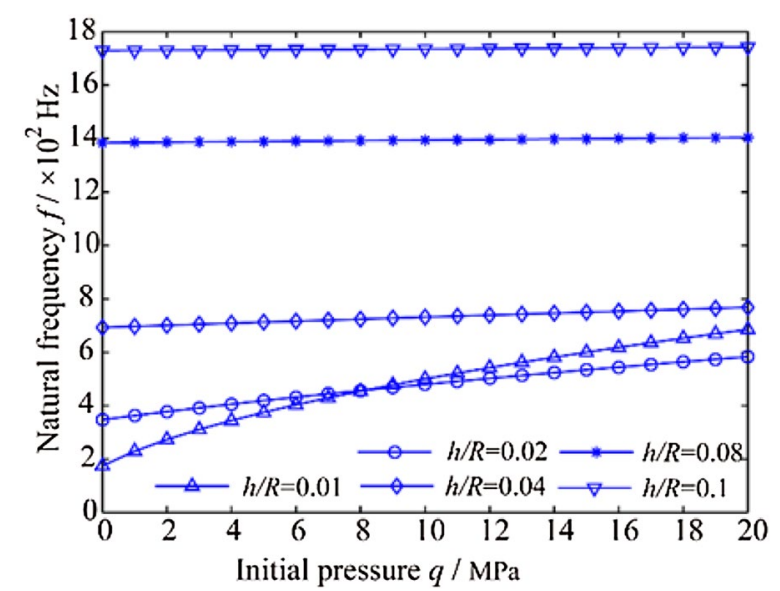

Figure 3 Variation of the natural frequency against $q$ for several special values of $h / R$

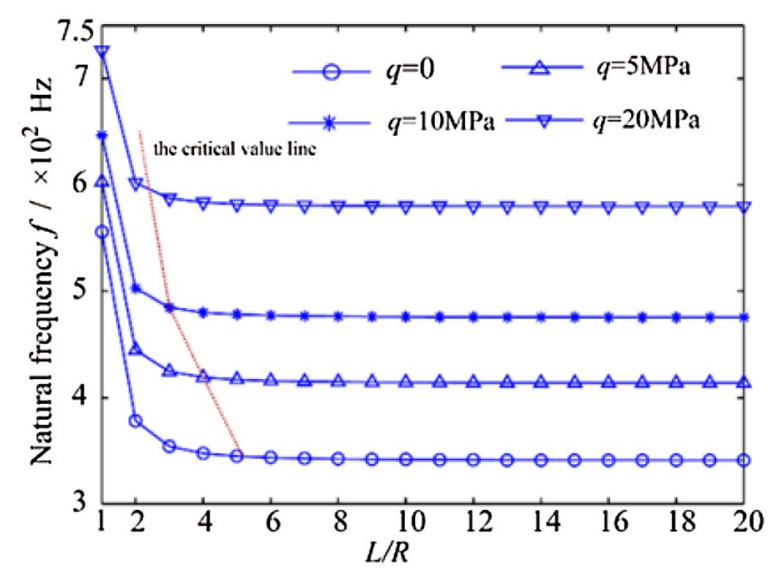

Figure 4 Variation of the natural frequency against $L / R$ ratio for several uniformly distributed pressures

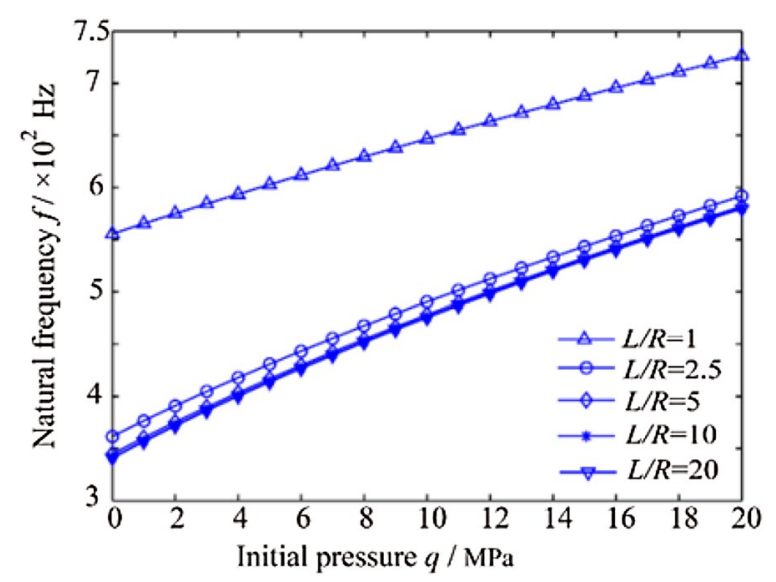

Figure 5 Variation of the natural frequency against $q$ for several special values of $L / R$ increases. The downtrend of natural frequency is much obvious when $L / R$ ratio is less than a certain extent and when $L / R$ increases to the value, the curve of natural frequency gradually flattens out. As demonstrated, if $q=0$, when $L / R \leq 5$, the natural frequency sharply decreases as $L / R$ ratio increases, and when $L / R>5$, the trend gradually flattens out. If $q=5 \mathrm{MPa}$, when $L / R \leq 4$, the natural frequency sharply decreases as $L / R$ ratio increases, and when $L / R>4$, the trend gradually flattens out. If $q=10 \mathrm{MPa}$, when $L / R \leq 3$, the natural frequency sharply decreases as $L / R$ ratio increases, and when $L / R>3$, the trend gradually flattens out. If $q=20 \mathrm{MPa}$, when $L / R \leq 2.5$, the natural frequency sharply decreases as $L / R$ ratio increases, and when $L / R>2.5$, the trend gradually flattens out. These all express that, for the curve of variation of natural frequency, there is a critical value before it slows down and the point of the critical value moves to the left as $q$ increases, and the moving amplitude also slowly becomes smaller as $q$ increases.

Figure 5 shows the variation of the natural frequency of the pipe against $q$ for several special $h / R$ ratio. As shown, for a certain $L / R$ ratio, the natural frequency always increases as the value of $q$ increases, which is mainly determined by $h / R$ ratio. However, for different values of $L / R$, the curve of natural frequency will integrally move up and down in the picture. And when $L / R \geq 5$, the curves tend to overlap. Therefore, $L / R$ ratio also determines the range of natural frequencies, and the critical value has an important reference significance for the design of the pipe.

\subsection{Influence of Pressure and Circumferential Wave Number on Natural Frequencies}

The natural frequencies shown in Figures 6 and 7 were calculated for $m=1, L / R=4$, and $h / R=0.02$.

Figure 6 shows the variation of the natural frequency of the pipe against the circumferential wave number $(n)$ with uniformly distributed pressure $(q)$. As shown, the natural frequency initially decreases and then increases as the value of $n$ increases. When $q=0$, the lowest point of the curve appears at $n=3$. When $q$ increases to a certain extent, the point will be at $n=2$. However, the minimum value of natural frequency always increases as $q$ increases.

Figure 7 shows the variation of the natural frequency of the pipe against $q$ for several special $n$. When $n=1$, the curve of natural frequency is a horizontal straight line, so $q$ has no effect on the natural frequency. When $n>1$, the natural frequency increases as the value of $q$ increases. And the trend of increasing is more and more obvious as $n$ increases. Therefore, for a short thin wall pipe, when the pressure is increasing the value of the circumferential 


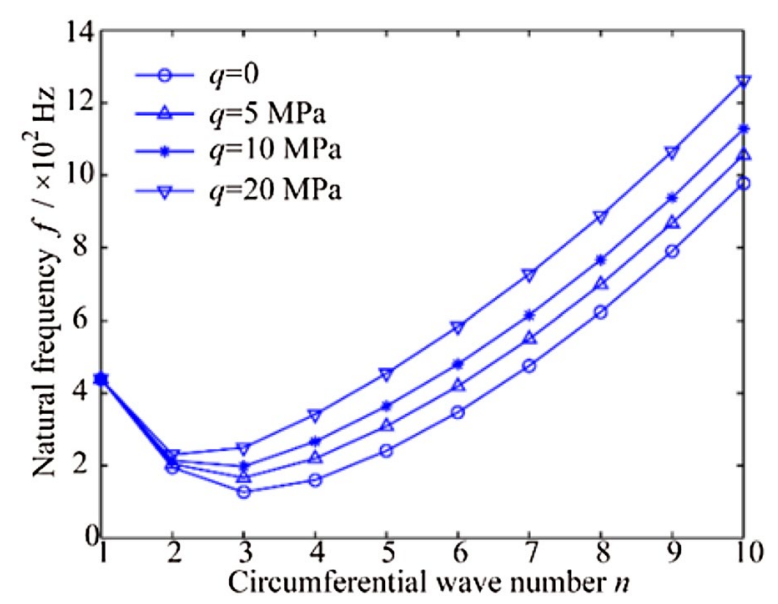

Figure 6 Variation of the natural frequency against $n$ for several uniformly distributed pressures

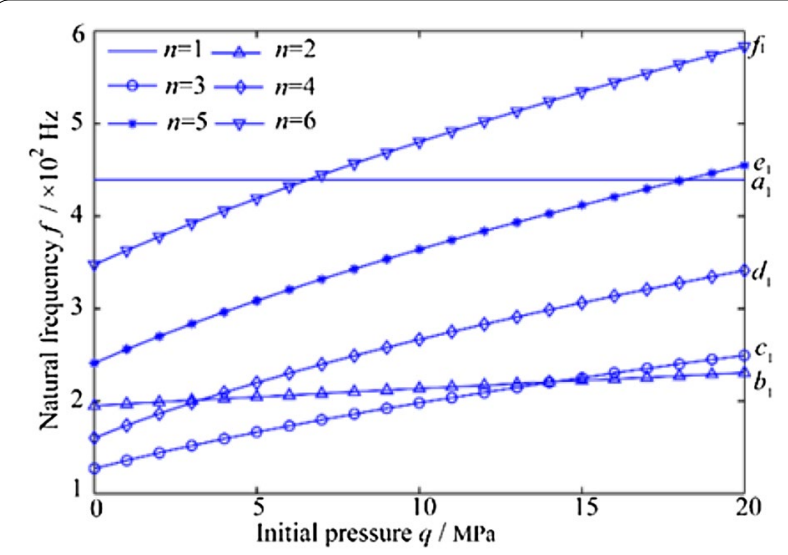

Figure $\mathbf{7}$ Variation of the natural frequency against $q$ for several special values of $n$

wave number directly determines the value of corresponding natural frequency. In addition, Figure 7 shows that when $q<14 \mathrm{MPa}$, the minimum natural frequency is at $n=3$ and when $q>14 \mathrm{MPa}$, the minimum value is at $n=2$. It accurately points out the critical value of $n$ corresponding to the minimum natural frequency. In the figure, $a_{1}, b_{1}, c_{1}, d_{1}, e_{1}$ and $f_{1}$ are the points of the value of natural frequencies when $q=20 \mathrm{MPa}$, which provide the reference for the next work.

\section{Validation, Analysis and Application Examples 5.1 Validation and Analysis}

To verify the results of the last part, the forced vibration of a pressure pipe with SS-SS boundary conditions was presented. The parameters: $L=2000 \mathrm{~mm}, h=10 \mathrm{~mm}$, $R=500 \mathrm{~mm}$ and $q=20 \mathrm{MPa}$, Pulse amplitude is $5 \%$, $E=2.06 \times 10^{11} \mathrm{~Pa}, \rho=7850 \mathrm{~kg} / \mathrm{m}^{3}$, and $\mu=0.3$. The natural frequencies for $i=2, j=6$ are listed in Table 2 .

The amplitudes of displacements in the $x, \theta$, and $z$ directions at position $(x / L=0.25, \theta=\pi / 4)$ of the neutral surface varying with the frequency $\omega(\mathrm{Hz})$ of internal pulsating loads are shown in Figure 8. It is seen from Figure 8 that some peak values exist at the points as $a_{1}$, $b_{1}, c_{1}, d_{1}, e_{1}, f_{1}, a_{2}, b_{2}, c_{2}, d_{2}, e_{2}$ and $f_{2}$ in the amplitudefrequency curves. These values correspond to the resonant responses of the pipe under internal pulsating loads. The frequencies are also corresponding to the natural frequencies listed in Table 2 and shown in Figure 7. So these all verify the validity of the former work. What's more, the large amplitude of the displacement at resonance points will be the main origin causing pipe broken.

\subsection{Application Examples}

Figure 9 shows two aspects of the common failure modes, for example, the long crack and the partial hole. Some selected mode shapes and frequencies of the pressure pipe with simply supported are computed and plotted in Figure 10. Though the deformation of the pressure pipe, it is helpful to understand certain features in the vibration characteristics of the pipe. From these mode shapes, it can be deduced that the long crack may be caused by the low-frequency vibration, which has a low axial wave number and a high circumferential wave number, and the partial hole may be caused by high-frequency vibration,

Table 2 Natural frequencies for $i=2$ and $j=6(\mathrm{~Hz})$

\begin{tabular}{lllllll}
\hline $\boldsymbol{m}$ & $\boldsymbol{n}$ & $\mathbf{3}$ & $\mathbf{4}$ & $\mathbf{5}$ & $\mathbf{6}$ \\
\cline { 2 - 6 } & $\mathbf{1}$ & $\mathbf{2}$ & $249.4\left(c_{1}\right)$ & $341.2\left(d_{1}\right)$ & $454.7\left(e_{1}\right)$ & $583.4\left(f_{1}\right)$ \\
1 & $439.4\left(a_{1}\right)$ & $230.5\left(b_{1}\right)$ & $406.0\left(c_{2}\right)$ & $402.9\left(d_{2}\right)$ & $483.6\left(e_{2}\right)$ & $601.8\left(f_{2}\right)$ \\
\hline
\end{tabular}




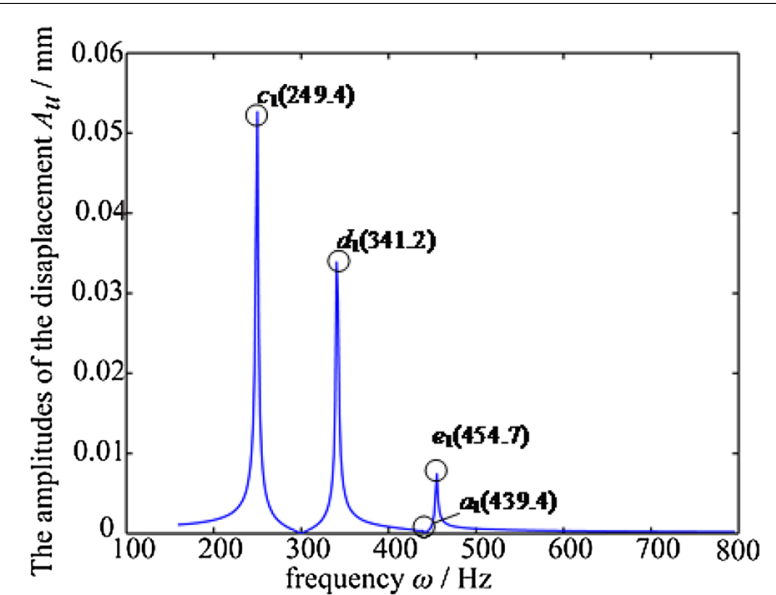

a In the $x$ direction

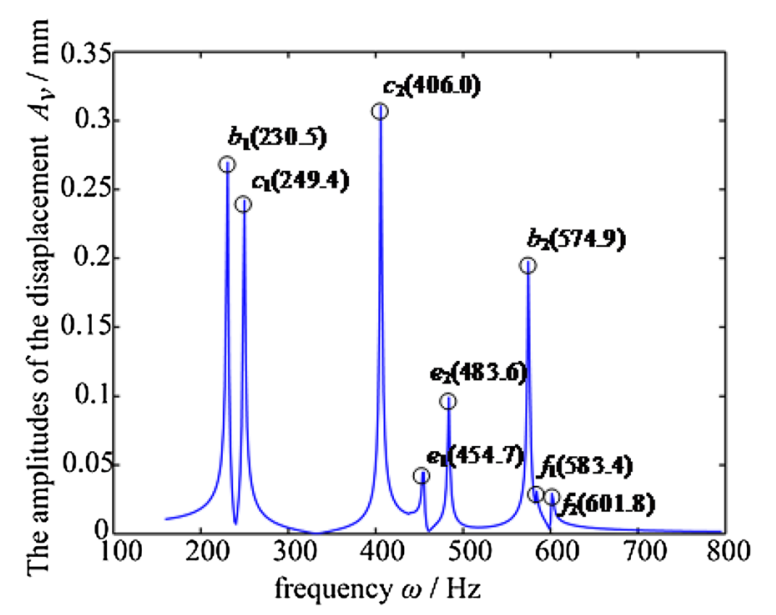

b In the $\theta$ direction

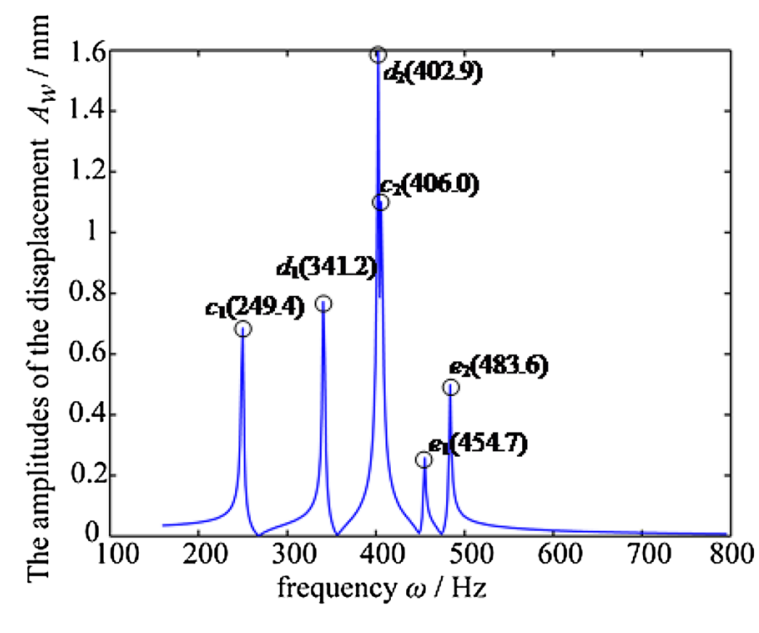

c In the $z$ direction.

Figure 8 Amplitudes of displacements in the $x, \theta$, and $z$ directions

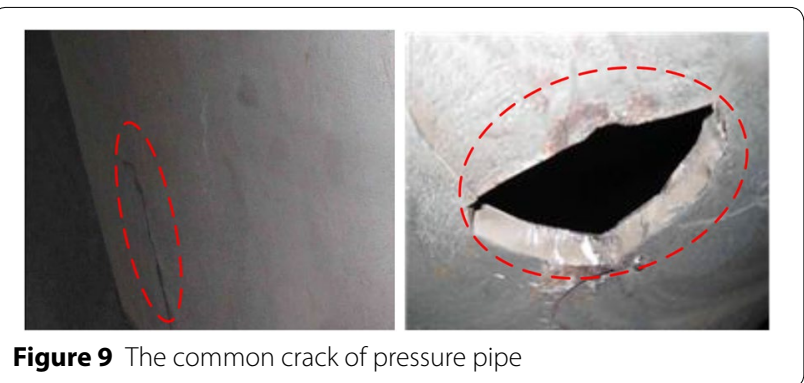

which has a high axial wave number and a high circumferential wave number.

\section{Conclusions}

Based on Sanders' shell theory, the vibration differential equation of the pipe with SS-SS boundary conditions was established by using the energy method, and the natural frequencies were obtained by solving the eigenvalue problem. The effects of pressure and geometric parameters on natural frequencies were analyzed. Some selected mode shapes and frequencies of the pressure pipe with simply supported were computed. Conclusions are as follows:

(1) For the compressor pipe, the value of distributed pressure impacts natural characteristics. Natural frequency increases as the pressure increases and the increasing value for higher mode is larger than that for lower modes. But the effect of distributed initial pressure on natural frequency for different circumferential wave numbers also abides by a certain rule of $n$. For a pipe with a constant $L / R$ and $h / R$ and $m=1$, the circumferential wave number according to the minimum natural frequency is determined by pressure. When $q$ is less than a certain threshold, $n=2$; otherwise, $n=3$. The rule also has a signification on the pipe dynamic design in the early design stage.

(2) $L / R$ and $h / R$ affect the sensitivity of pressures on the natural frequency of pipe. Generally, the thinner the pipe is, the stronger the sensitivity is. And when $L / R$ is greater than a certain threshold, the effect of pressure on natural frequency would not be obvious. So, more attention should be paid to these critical values of $L / R$ and $h / R$ when designing pipe.

(3) Pulse frequency close to the natural frequency of pipe may cause resonance, which may lead to pipe 

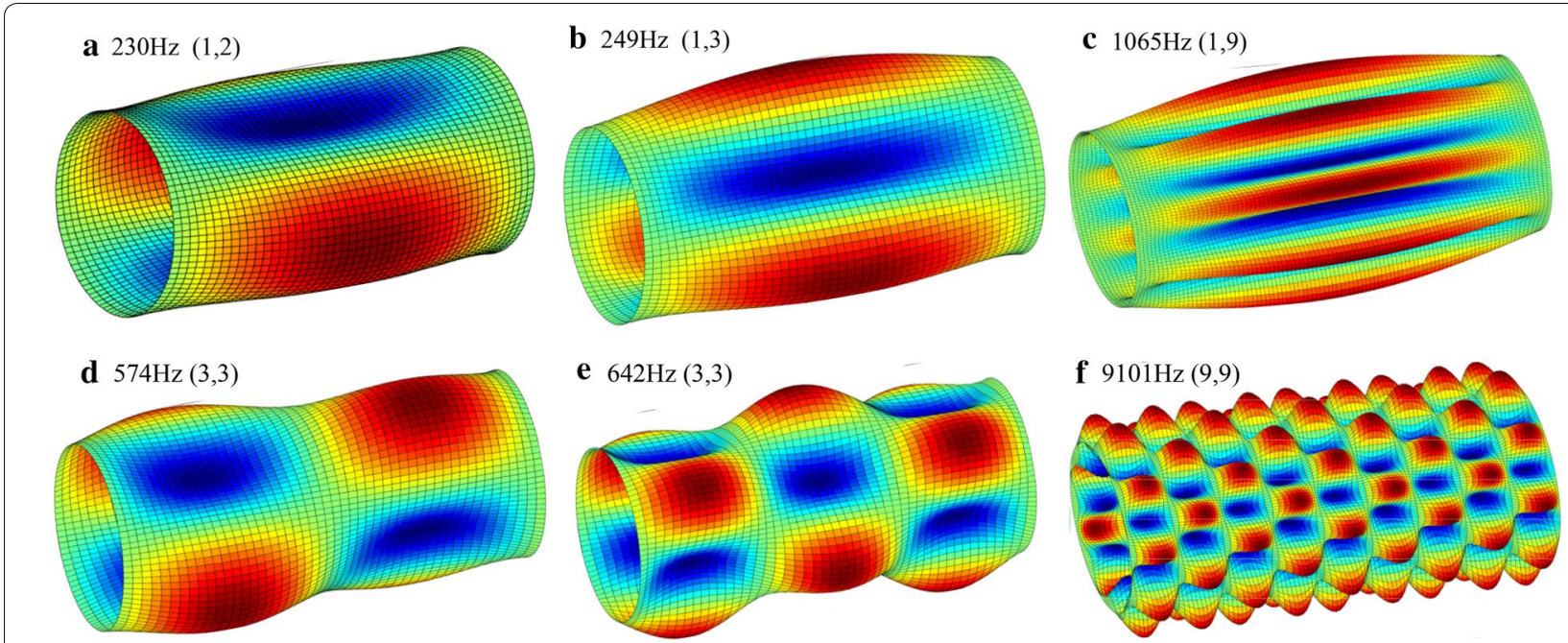

Figure 10 The modal shapes of pressure pipe with simply supported

broken. From the failure modes of pressure pipe, the long crack may be caused by the low-frequency vibration, which has a low axial half-wave number and a high circumferential wave number, and the partial hole may be caused by high-frequency vibration, which has a high axial half-wave number and a high circumferential wave number. Knowing these facts is important for pipe dynamic design and avoiding resonance points.

\section{Authors' Contributions}

L-CF was in charge of the theory modelling and the whole structure of articles; T-QS and S-HX assisted with simulation and analysis; W-BC make some significant advice. All authors read and approved the final manuscript.

\section{Author Details}

${ }^{1}$ School of Mechanical Engineering \& Automation, Northeastern University, Shenyang 110819, China. ${ }^{2}$ Key Laboratory of Vibration and Control of Aero-propulsion Systems, Northeastern University, Shenyang 110819, China.

\section{Authors' Information}

Chao-Feng Li, born in 1980, Ph.D., is currently an associate professor at School of Mechanical Engineering \& Automation, Northeastern University, China, in 2016. His research interests include mechanical dynamics and control.

Qian-Sheng Tang, born in 1989, is current a Ph.D. candidate at School of Mechanical Engineering \& Automation, Northeastern University, China.

Hou-Xin She, born in 1991, is current a Ph.D. candidate at School of Mechanical Engineering \& Automation, Northeastern University, China.

Bang-Chun Wen, born in 1930, is a Professor School of Mechanical Engineering \& Automation, Northeastern University, China. His research interests include vibration utilization engineering.

\section{Competing interests}

The authors declare that they have no competing interests.

\section{Funding}

Supported by National Natural Science Foundation of China (Grant No. 51575093), Fundamental Research Funds for the Central Universities of China (Grant Nos. N160313001, N170308028).

\section{Appendix}

$$
\begin{aligned}
& \boldsymbol{M}_{1}= \rho h \int_{0}^{1} \int_{0}^{2 \pi} \boldsymbol{U} \boldsymbol{U}^{\mathrm{T}} R L \mathrm{~d} \theta \mathrm{d} \xi \\
& \boldsymbol{M}_{2}=\rho h \int_{0}^{1} \int_{0}^{2 \pi} \boldsymbol{V} \boldsymbol{V}^{\mathrm{T}} R L \mathrm{~d} \theta \mathrm{d} \xi, \\
& \boldsymbol{M}_{3}=\rho h \int_{0}^{1} \int_{0}^{2 \pi} \boldsymbol{W} \boldsymbol{W}^{\mathrm{T}} R L \mathrm{~d} \theta \mathrm{d} \xi, \\
& \boldsymbol{K}_{1}=\int_{0}^{1} \int_{0}^{2 \pi}\left\{\frac{E h}{\left(1-\mu^{2}\right)} \frac{1}{L^{2}} \frac{\partial \boldsymbol{U}}{\partial \xi} \frac{\partial \boldsymbol{U}^{\mathrm{T}}}{\partial \xi}+\left[\frac{E h}{\left(1-\mu^{2}\right)} \frac{1-\mu}{2} \frac{1}{R^{2}}\right.\right. \\
&\left.\left.+\frac{E h^{3}}{12\left(1-\mu^{2}\right)} \frac{(1-\mu)}{2 R^{2}} \frac{1}{4 R^{2}}\right] \frac{\partial \boldsymbol{U}}{\partial \theta} \frac{\partial \boldsymbol{U}^{\mathrm{T}}}{\partial \theta}\right\} R L \mathrm{~d} \theta \mathrm{d} \xi,
\end{aligned}
$$$$
\boldsymbol{K}_{2}=\int_{0}^{1} \int_{0}^{2 \pi}\left\{\frac{E h}{\left(1-\mu^{2}\right)} \frac{2 \mu}{R L} \frac{\partial \boldsymbol{U}}{\partial \xi} \frac{\partial \boldsymbol{V}^{\mathrm{T}}}{\partial \theta}+\left[\frac{E h}{\left(1-\mu^{2}\right)} \frac{1-\mu}{2} \frac{2}{R L}\right.\right.
$$$$
\left.\left.-\frac{E h^{3}}{12\left(1-\mu^{2}\right)} \frac{(1-\mu)}{2 R^{2}} \frac{3}{2 R L}\right] \frac{\partial \boldsymbol{U}}{\partial \theta} \frac{\partial \boldsymbol{V}^{\mathrm{T}}}{\partial \xi}\right\} R L \mathrm{~d} \theta \mathrm{d} \xi,
$$ 


$$
\begin{aligned}
& \boldsymbol{K}_{3}=\int_{0}^{1} \int_{0}^{2 \pi}\left\{\frac{E h}{\left(1-\mu^{2}\right)} \frac{2 \mu}{R L} \frac{\partial \boldsymbol{U}}{\partial \xi} \boldsymbol{W}^{\mathrm{T}}\right. \\
& \left.+\frac{E h^{3}}{12\left(1-\mu^{2}\right)} \frac{(1-\mu)}{2 R^{2}} \frac{2}{R L} \frac{\partial \boldsymbol{U}}{\partial \theta} \frac{\partial^{2} \boldsymbol{W}^{\mathrm{T}}}{\partial \xi \partial \theta}\right\} R L \mathrm{~d} \theta \mathrm{d} \xi, \\
& \boldsymbol{K}_{4}=\int_{0}^{1} \int_{0}^{2 \pi}\left\{\left[\frac{E h}{\left(1-\mu^{2}\right) R^{2}}+\frac{E h^{3}}{12\left(1-\mu^{2}\right) R^{4}}\right] \frac{\partial \boldsymbol{V}}{\partial \theta} \frac{\partial \boldsymbol{V}^{\mathrm{T}}}{\partial \theta}\right. \\
& \left.+\left[\frac{E h}{\left(1-\mu^{2}\right)} \frac{1-\mu}{2 L^{2}}+\frac{9 E h^{3}}{12\left(1-\mu^{2}\right)} \frac{(1-\mu)}{8 R^{2} L^{2}}\right] \frac{\partial V}{\partial \xi} \frac{\partial V^{\mathrm{T}}}{\partial \xi}\right\} R L \mathrm{~d} \theta \mathrm{d} \xi, \\
& \boldsymbol{K}_{5}=\int_{0}^{1} \int_{0}^{2 \pi}\left\{\frac{E h}{\left(1-\mu^{2}\right)} \frac{2}{R^{2}} \frac{\partial \boldsymbol{V}}{\partial \theta} \boldsymbol{W}^{\mathrm{T}}-\frac{E h^{3}}{12\left(1-\mu^{2}\right)} \frac{2 \mu}{R^{2} L^{2}} \frac{\partial \boldsymbol{V}}{\partial \theta} \frac{\partial^{2} \boldsymbol{W}^{\mathrm{T}}}{\partial \xi^{2}}\right. \\
& \left.-\frac{2 E h^{3}}{12\left(1-\mu^{2}\right) R^{4}} \frac{\partial \boldsymbol{V}}{\partial \theta} \frac{\partial^{2} \boldsymbol{W}^{\mathrm{T}}}{\partial \theta^{2}}-\frac{3 E h^{3}}{12\left(1-\mu^{2}\right)} \frac{(1-\mu)}{R^{2} L^{2}} \frac{\partial \boldsymbol{V}}{\partial \xi} \frac{\partial^{2} \boldsymbol{W}^{\mathrm{T}}}{\partial \xi \partial \theta}\right\} \\
& \times R L \mathrm{~d} \theta \mathrm{d} \xi, \\
& \boldsymbol{K}_{6}=\int_{0}^{1} \int_{0}^{2 \pi}\left\{\frac{E h}{\left(1-\mu^{2}\right)} \frac{1}{R^{2}} W W^{\mathrm{T}}+\frac{E h^{3}}{12\left(1-\mu^{2}\right)} \frac{1}{L^{4}} \frac{\partial^{2} W}{\partial \xi^{2}} \frac{\partial^{2} W^{\mathrm{T}}}{\partial \xi^{2}}\right. \\
& +\frac{E h^{3}}{12\left(1-\mu^{2}\right)} \frac{2 \mu}{R^{2} L^{2}} \frac{\partial^{2} W}{\partial \xi^{2}} \frac{\partial^{2} W^{\mathrm{T}}}{\partial \theta^{2}}+\frac{E h^{3}}{12\left(1-\mu^{2}\right)} \frac{1}{R^{4}} \frac{\partial^{2} W}{\partial \theta^{2}} \frac{\partial^{2} W^{\mathrm{T}}}{\partial \theta^{2}} \\
& \left.+\frac{E h^{3}}{12\left(1-\mu^{2}\right)} \frac{(1-\mu)}{2 R^{2}} \frac{4}{L^{2}} \frac{\partial^{2} W}{\partial \xi \partial \theta} \frac{\partial^{2} W^{\mathrm{T}}}{\partial \xi \partial \theta}\right\} R L \mathrm{~d} \theta \mathrm{d} \xi, \\
& \boldsymbol{H}=-\int_{0}^{1} \int_{0}^{2 \pi} \frac{q L}{2}\left[\left(\frac{\partial^{2} \boldsymbol{W}}{\partial \theta^{2}}+\boldsymbol{W}\right) \boldsymbol{W}^{\mathrm{T}}\right] \mathrm{d} \theta \mathrm{d} \xi .
\end{aligned}
$$

\section{Publisher's Note}

Springer Nature remains neutral with regard to jurisdictional claims in published maps and institutional affiliations.

Received: 17 October 2017 Accepted: 29 October 2018

Published online: 14 November 2018

\section{References}

[1] Tingyue Hao, Guiqing Chen, Guangyao Xu. Study of vibration of pipe conveying fluid. Water Conservancy Science and Technology and Economy, 2008, 14(7): 532-534. (in Chinese)

[2] M Kheiri, M P Paidoussis, M Amabili, et al. Three-dimensional dynamics of long pipes towed underwater Part 1: The equations of motion. Ocean Engineering, 2013, 64: 153-160.

[3] M Kheiri, M P Paidoussis, M Amabili, et al. Three-dimensional dynamics of long pipes towed underwater Part 2 Linear dynamics. Ocean Engineering, 2013, 64: 161-173.
[4] M H Ghayesh, M P Paidoussis, M Amabili. Nonlinear dynamics of cantilevered extensible pipes conveying fluid. Journal of Sound and Vibration, 2013, 332: 6405-6418.

[5] KYamashita, H Furuya, H Yabuno, et al. Nonplanar vibration of a vertical fluid-conveying pipe (Effect of horizontal excitation at the upper end). Journal of Vibration and Acoustics-Transactions of the ASME, 2014: 136.

[6] Yanlei Zhang, Liqun Chen. Internal resonance of pipes conveying fluid in the supercritical regime. Nonlinear Dynamics, 2012, 67: 1505-1514.

[7] Yanlei Zhang, Liqun Chen. External and internal resonances of the pipe conveying fluid in the supercritical regime. Journal of Sound and Vibration, 2013, 332: 2318-2337.

[8] M S Qatu. Recent research advances in the dynamic behavior of shells: 1989-2000, Part 2: Homogeneous shells. Applied Mechanics Reviews, 2002, 55(5): 415-434

[9] A W Leissa. Vibration of shells. Washington, DC, USA: Scientific and Technical Information Office, National Aeronautics and Space Administration, 1973

[10] C T Loy, KY Lam, C Shu. Analysis of cylindrical shells using generalized differential quadrature. Shock and Vibration, 1997, 4(3): 193-198.

[11] H Li, KY Lam. Frequency characteristics of a thin rotating cylindrical shell using the generalized differential quadrature method. International Journal of Mechanical Sciences, 1998, 40(5): 443-459.

[12] CT Loy, KY Lam, J N Reddy. Vibration of functionally graded cylindrical shells. International Journal of Mechanical Sciences, 1999, 41(3): 309-324.

[13] S C Pradhan, C T Loy, KY Lam, et al. Vibration characteristics of functionally graded cylindrical shells under various boundary conditions. Applied Acoustics, 2000, 61(1): 111-129.

[14] M J Ebrahimi, M M Najafizadeh. Free vibration analysis of two-dimensional functionally graded cylindrical shells. Applied Mathematical Modelling, 2014, 38(1): 308-324.

[15] Xiang Xie, Guoyong Jin, Zhigang Liu. Free vibration analysis of cylindrical shells using the Haar wavelet method. International Journal of Mechanical Sciences, 2013, 77: 47-56.

[16] Guanmo Xie. Free vibration analysis of circular cylindrical shells using transfer matrix method. 2011 International Conference on IEEE, Wuhan, China, 2011: 423-426.

[17] Lu Dai, Tiejun Yang, Jingtao Du, et al. An exact series solution for the vibration analysis of cylindrical shells with arbitrary boundary conditions. Applied Acoustics, 2013, 74(3): 440-449.

[18] Shupeng Sun, Dengqing Cao, Qingkai Han. Vibration studies of rotating cylindrical shells with arbitrary edges using characteristic orthogonal polynomials in the Rayleigh-Ritz method. International Journal of Mechanical Sciences, 2013, 68: 180-189.

[19] M R Isvandzibaei, H Jamaluddin, R I Raja Hamzah. Frequency analysis of multiple layered cylindrical shells under lateral pressure with asymmetric boundary conditions. Chinese Journal of Mechanical Engineering, 2014, 27: 23-31.

[20] M R Isvandzibaei, H Jamaluddin, R I Raja Hamzah. Natural frequency characteristics of thin-walled homogeneous and manifold layered cylindrical shells under pressure using energy method. Journal of Central South University, 2014, 21: 521-532.

\section{Submit your manuscript to a SpringerOpen ${ }^{\circ}$ journal and benefit from:}

- Convenient online submission

$\checkmark$ Rigorous peer review

- Open access: articles freely available online

- High visibility within the field

- Retaining the copyright to your article

Submit your next manuscript at $>$ springeropen.com 